\title{
Biss einer Ammendornfingerspinne - eine zukünftig häufiger zu stellende Diagnose?
}

\author{
Bites of Yellow Sack Spider - More Frequently to be Seen in the Future?
}

Autoren

Institut

\author{
S. Weimann, T. Vogt, C. Pföhler
}

Klinik für Dermatologie, Venerologie und Allergologie, Universitätsklinikum des Saarlandes, Homburg/Saar

\section{Bibliografie}

DOI http://dx.doi.org/

10.1055/s-0030-1256098

Akt Dermatol 2011; 37:

22-23 @ Georg Thieme

Verlag KG Stuttgart · New York ISSN 0340-2541

\section{Korrespondenzadresse}

\section{Stefanie Weimann}

Klinik für Dermatologie, Venerologie und Allergologie Universitätsklinikum des Saarlandes

Kirrbergerstr. 1 - Gebäude 18 66421 Homburg stefanie.weimann@uks.eu

\section{Zusammenfassung \\ $\nabla$}

Mit Fortschreiten der Klimaerwärmung werden auch deutsche Dermatologen mit neuen Insekten

\section{Kasuistik}

$\nabla$

Ein 46-jähriger Patient war am Tag zuvor mittags am Hals lateral von einer Spinne gebissen worden, bei Grünarbeiten auf dem Campus. Zunächst habe sich eine lokale Schwellung und Rötung gezeigt. Bei Zunahme der Allgemeinsymptomatik mit Atemnot, Schluckbeschwerden sowie einer starken Müdigkeit erfolgte die notfallmäßige Vorstellung in unserer Ambulanz am Morgen darauf. Am Hals seitlich rechts fand sich eine infiltrierte, urtikariell imponierende, überwärmte Hautrötung. Zentral war eine punktuelle weißliche BissStelle sichtbar ( $\bullet$ Abb. 1 und $\mathbf{2}$ ).

Der Patient konnte das Aussehen der Spinne detailliert beschreiben; die Spinne konnte dann durch einen insektenkundigen Kollegen der Familie der Dornfingerspinnen (Miturgidae) zugeordnet und über geeignete Fotografien genauer als Spinne aus der Gattung Dornfinger (Cheiracanthium) und Vertreter der Art „Ammendornfinger“ eindeutig bestimmt werden.

Die Akuttherapie bestand in der Gabe von Methylprednisolon $64 \mathrm{mg} / \mathrm{d}$ i.v. sowie einer externen Therapie mit einer Betamethason-haltigen Lotio. Hierunter kam es zu einer Abblassung des Erythems im Verlauf von 24 Stunden. Sämtliche Vital- und Laborparameter (kleines und Diff.-BB, Herzenzyme, Gerinnung, Infektparameter) zeigten sich in der Überwachung unauffällig. Es kam bei unserem Patienten nicht zur Entwicklung einer sonst durchaus möglichen Nekrose an der Biss-Stelle. und neuen Krankheiten konfrontiert. Hier beschreiben wir einen klinisch eindrucksvollen Fall des Bisses einer Ammendornfingerspinne (Cheiracanthium punctorium).

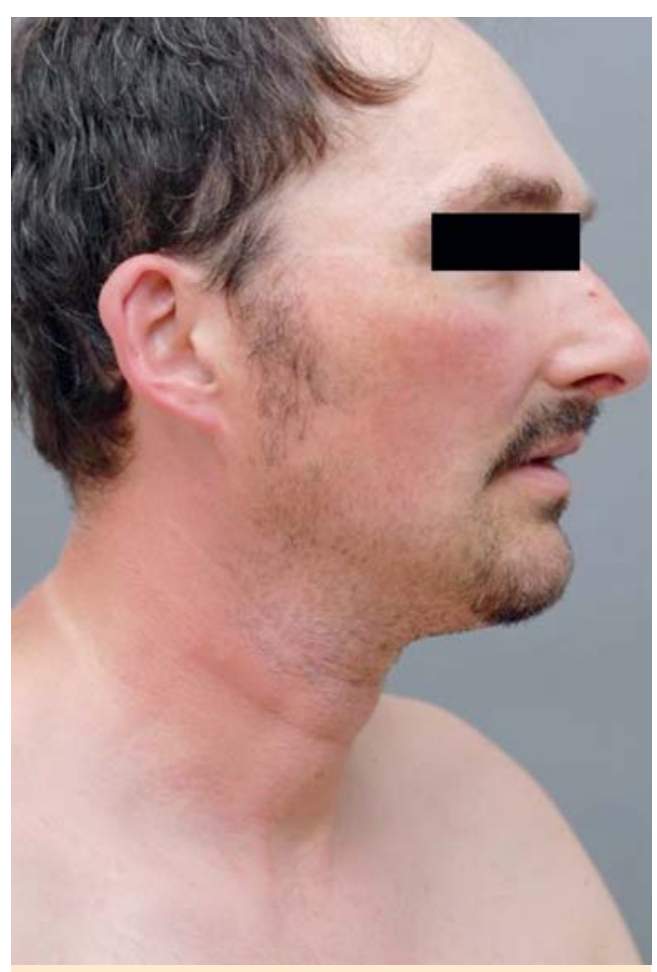

Abb. 1 Biss-Stelle am Hals lateral rechts.

\section{Diskussion}

In tropischen Ländern stellen neben den Giftschlangen Spinnenbisse eine nicht unbedeutende Gefahr für den Menschen dar [1]. In Deutschland ist dies jedoch ein seltenes Ereignis. Die aus dem Mittelmeerraum stammende Ammendornfingerspinne (Cheiracanthium punctorium) kommt seit einigen Jahren auch nördlich der Alpen vor. Der Klimawandel begünstigt die Wanderung der 


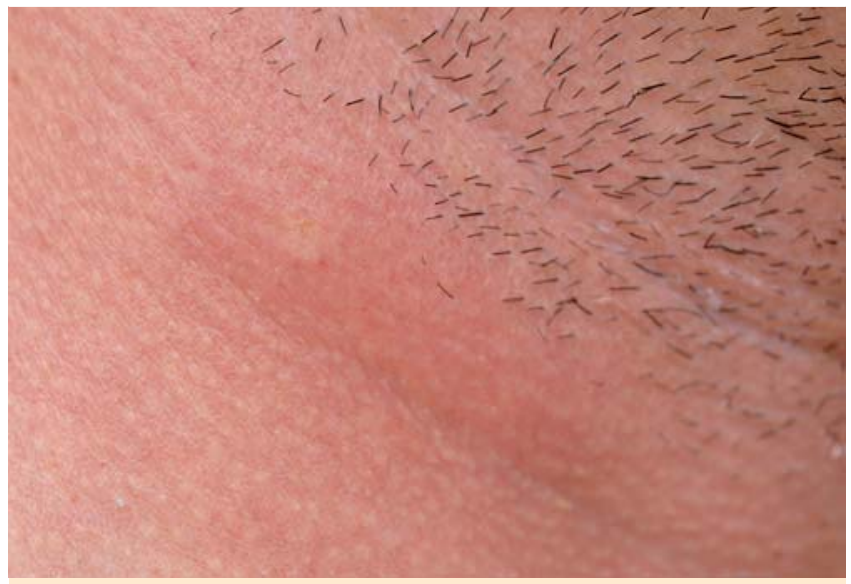

Abb. 2 Lokalbefund etwa $16 \mathrm{~h}$ nach Biss: urtikarielle, infiltrierte, überwärmte Hautrötung.

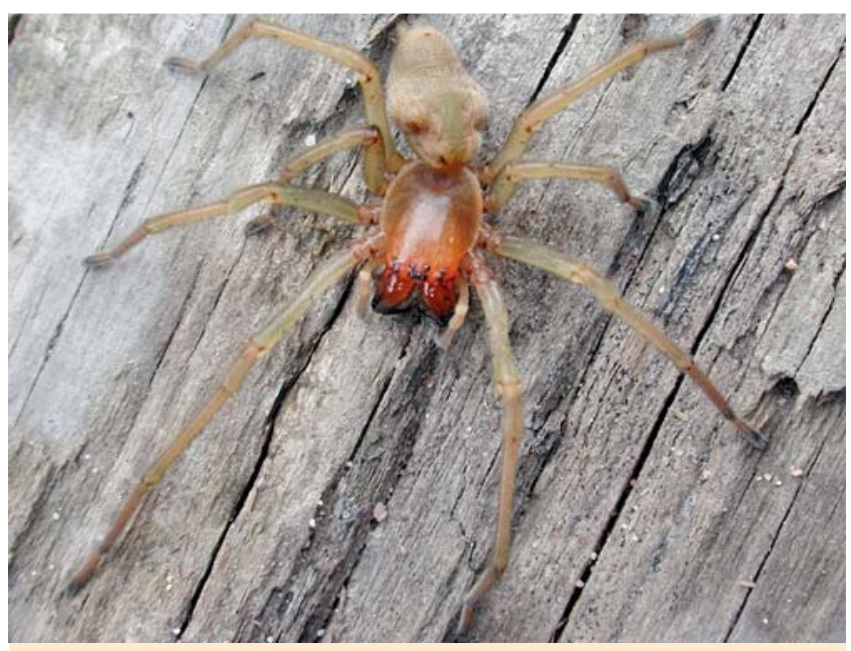

Abb. 3 Ammendornfingerspinne.

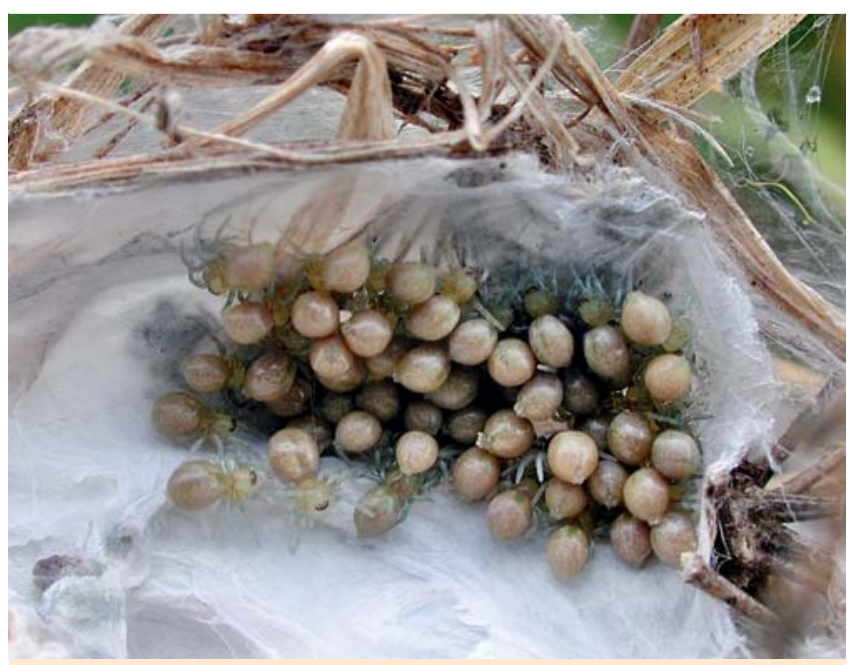

Abb. 4 Eikokon im Brutgespinst.

Spinne Richtung Norden. Im Saarland wurde die Spinne bereits 1983 mehrfach nachgewiesen [2]. Bevorzugte Lebensräume sind Wiesen mit hohem Gras. Die 1,2 bis 1,6 cm große Spinne hat eine blass-grünliche Grundfarbe und auf dem Hinterleib eine rötliche Zeichnung. Der Kopfschild ist kräftig orange-braun gefärbt, die
Kieferklauen sind auffallend stark ausgeprägt. Am Ende der Beine und Fühlertaster sind charakteristische schwarze Punkte sichtbar. Die relativ kleinen Augen sind in zwei dicht hintereinander liegenden Doppelreihen angeordnet ( Abb. 3).

Die Ammendornfingerspinne gilt als die giftigste Spinne Mitteleuropas. Zwar ist die Giftwirkung anderer Spinnen ähnlich stark, z.B. in Südeuropa die der Tarantel, der Schwarzen Witwe oder Wolfsspinne, allerdings besitzt nur die Dornfingerspinne so starke Kieferklauen, dass sie die menschliche Haut durchbeißen kann. Die Ammendornfingerspinne baut typischerweise keine Fangnetze und jagt nachts. Zu Bissen kommt es meist im Monat August, da um diese Zeit die Eiablage in das Brutgespinst erfolgt ( Abb.4), das von den Weibchen intensiv bewacht und durch Bisse verteidigt wird.

Die Symptome nach einem Stich ähneln in der Regel denen eines Wespen- oder Bienenstichs. Das Nervengift der Spinne führt jedoch zu besonders starken Schmerzen. Es wirkt ausgeprägt gewebeschädigend mit starker, akuter Schwellung. In der Literatur werden auch immer wieder Nekrosen an der Biss-Stelle beschrieben [3]. Nach einem Biss sollte die Wunde, ganz entgegen den üblichen Praktiken, zunächst mit Wärme und erst später mit kalten Umschlägen behandelt werden. Die initiale Wärmeapplikation kann das wärmeinstabile Spinnengift teilweise inaktivieren, zudem wird durch die Gewährleistung einer guten Mikrozirkulation der Entstehung einer lokalen Nekrose entgegengewirkt. Selten sind schwere Verläufe mit Schüttelfrost, Schwindel, Erbrechen, leichtem Fieber oder Kreislaufkollaps beschrieben. Nach 24-30 Stunden sind die Symptome meist vollständig abgeklungen. Todesfälle sind bislang nicht beschrieben $[4,5]$.

Gesicherte Angaben zur Häufigkeit von Bissen existieren nicht. Oft bleibt unklar, welches Tier den Biss verursachte, da die Spinne meist nicht zur Bestimmung vorliegt oder gar nicht gesehen wurde. Auch wenn Ammendornfingerspinnen-Bisse in Deutschland sehr selten vorkommen, so sollten Dermatologen doch mit einer möglichen Symptomatik und der notwendigen Therapie vertraut sein.

Wir danken Herrn Volker Fässler (Pflegedienst) für die exzellenten Fotos einer Ammendornfingerspinne.

\section{Abstract}

\section{Bites of Yellow Sack Spider - More Frequently to be Seen in the Future? \\ $\nabla$}

As a consequence of global warming also German dermatologists must become aware of „new“ insects and insect-caused diseases. Here we describe the clinically impressing bite-reaction caused by the Cheiracanthium punctorium-spider.

\section{Literatur}

1 Habermehl G, Mebs D. Spinnenbisse in Deutschland. Dtsch Med Wschr 1979; 104: 681-682

2 Schichtel M. Cheiracanthium (Chiranthium) punctorium (VILL.) im südlichen Saarland. Faun-flor Not Saarl 1983; 15: 201 - 202

3 Vetter RS, Isbister GK, Bush SP, Boutin LJ. Verified bites by yellow sac spiders (genus Cheiracanthium) in the united states and America: where is the necrosis? Am J Trop Med Hyg 2006; 74 (6): 1043 - 1048

4 Bellmann $H$. Kosmos Atlas Spinnentiere Europas. 3. Aufl. Stuttgart: Kosmos; 2006

5 Wolf A. Cheiracanthium punctorium - Portrait einer berüchtigten Spinne. Natur und Museum 1988; 118: 310 - 317 